\title{
Use of rep-PCR to define genetic relatedness among Bacteroides fragilis strains
}

\author{
S. R. MORAES, R. B. GONÇALVES*, C. MOUTONं, L. SELDIN, M. C. S. FERREIRA and \\ R. M. C. P. DOMINGUES
}

Instituto de Microbiologia Prof. Paulo de Góes, UFRJ, Rio de J aneiro, Brasil *Faculdade de Odontologia de Piracicaba/UNICAMP, Brasil and †Faculté de Medicine Dentaire, Universite Laval, Quebec, Canada

\begin{abstract}
Bacteroides fragilis, a component of the normal flora and an important anaerobic pathogen in non-intestinal endogenous infections, has recently been associated with enteric diseases. In this study, $41 \mathrm{~B}$. fragilis strains were analysed in relation to their genetic diversity. This collection included two reference strains (ATCC 23745 and 25285), 20 isolates from non-intestinal infections, six from intestinal infections, five from intestinal microflora and eight from an aquatic environment. The fingerprints were gener ated by using two repetitive sequences (REP and ERIC) as primers to PCR (repP(R). A dendrogram was obtained with the Taxotron (B) Program. Three clusters (threshold genotypes I, II and III) were observed when the genetic distance was 0.30 . These results confirm previous data found regarding the genotypical diversity of $B$. fragilis.
\end{abstract}

\section{Introduction}

Bacteroides fragilis, an obligatory anaerobic bacterial component of the normal flora, is an important pathogen in endogenous infections such as intraabdominal abscess and septicaemia [1,2]. In recent years, several studies have focused on the involvement of $B$. fragilis in infections located in the mucosa, causing, for example, diarrhoea $[3,4]$.

In infectious diseases where the probable aetiological agent is a member of the normal flora (as is B. fragilis), simple species identification is not useful for distinguishing between infection and colonisation. In this context, clinical microbiologists have attempted to develop procedures that provide more detailed intraspecies delineation.

Several methods for detecting phenotypic or genotypic variation have been used to characterise bacterial isolates and to discriminate strains that are potential pathogens from commensal strains; however, only a few approaches have been applied to B. fragilis. Serotyping demonstrated a wide heterogeneity of capsular antigens among B. fragilis strains [5]. Genotypic methods, such

Received 17 Feb. 1999; revised version received 10 Aug. 1999; accepted 13 Aug. 1999.

Corresponding author: Professor R. M. C. P. Domingues (e-mail: IMMMREG@ microbio.UFRJ.br). as rRNA restriction fragment length polymorphism (RFLP) [6], restriction endonuclease analysis (REA) [7], ribotyping $[7,8]$ and arbitrary primed PCR (AP$P C R$ ) [9], have al so been used to study $B$. fragilis strains and a great diversity has been found. However, if different $B$. fragilis strains are associated with different diseases, many questions remain unanswered.

PCR-based DNA fingerprint methods have been used for molecular typing of several bacterial pathogens and have been cited as techniques that are universally applicable, rapid and with considerable discriminatory power $[10,11]$. PCR utilising interspersed repetitive elements as primers for the amplification of regions between neighbouring repetitive elements has been used (REP-PCR, ERIC-PCR and BOX-PCR) and has been collectively designated as rep-PCR [12-14].

This report describes the use of rep-PCR to assay $B$. fragilis strains from different sources (non-intestinal infections, intestinal infections, intestinal microflora and aquatic environment) to evaluate the biological and genotypic relatedness among them.

$M$ aterials and methods

Bacterial strains and growth conditions

A total of 41 B. fragilis strains was examined, including two type strains (ATCC 25285 and ATCC 
23745) obtained from the American Type Culture Collection, Rockville MD, USA. Thirty-one strains were isolated from different individuals (non-intestinal infections, 20; intestinal infections, 6; intestinal microflora, 5) and eight strains were isolated from an aquatic environment in Rio de J aneiro in the period 1980- 1997 (Table 1). Bacterial identification was by conventional bacteriological tests based on bile resistance, catalase production, a negative indole test and fermentation of sugars [15]. All strains were maintained in our culture collection, and were activated by growth in pre-reduced anaerobic sterilised brain-heart infusion broth (BHIPRAS) when required and then cultured in the same medium for $18 \mathrm{~h}$ at $37^{\circ} \mathrm{C}$ as a source of bacterial suspensions for the following analyses.

\section{DNA template preparation}

Cells from broth cultures $(5 \mathrm{ml})$ in the logarithmic phase were harvested by centrifugation at $13000 \mathrm{~g}$ for $1 \mathrm{~min}$. The individual cell pellets were stored at $-20^{\circ} \mathrm{C}$ without additional treatment until DNA isolation. Extraction and purification of chromosomal DNA was performed according to a modification of the procedure of Smith et al. [16], with the addition of RNAase treatment [17]. The concentration of DNA in the samples was determined by spectrophotometry with the GenQuant apparatus (Pharmacia).

\section{Primers}

Primer sequences corresponding to REP - REPIR-I (5'-IIIICGICGICATCIGGC-3') and REP2-I (5'-ICGI CTTATCIGGCCTAC-3') - and ERIC - ERICIR (5'ATGTAAGCTCCTGGGGATTCAC-3') and ERIC2 (5'AAGTAAGTGACTGGGGTGAGCG-3') [14] - were used to evaluate the amplicon profiles by PCR.

\section{Amplification conditions}

Fifty ng of DNA template were used in each reaction. The rep-PCR amplification was performed in a $25-\mu$ l volume containing $80 \mathrm{pmol}$ each of two opposing primers, $1.25 \mathrm{~mm}$ deoxynucleoside triphosphates and $2.5 \mathrm{U}$ of Taq polymerase. Temperature cycling was controlled in a model PTC-100-60 cycler (Peltin-Effect Cycling, Watertown, USA) programmed as follows: one initial cycle at $95^{\circ} \mathrm{C}$ for $7 \mathrm{~min} ; 30$ cycles of denaturation at $94^{\circ} \mathrm{C}$ for $1 \mathrm{~min}$, annealing at $44^{\circ}$ or $52^{\circ} \mathrm{C}$ for $1 \mathrm{~min}$ with REP and ERIC primers, respectively, and extension at $65^{\circ} \mathrm{C}$ for 2 min with a single final extension cycle at $65^{\circ} \mathrm{C}$ for $15 \mathrm{~min}$ and a final soak at $4^{\circ} \mathrm{C}$. PCR mixtures were overlaid with $25 \mu \mathrm{l}$ of mineral oil (M 3516; Sigma).

On completion of the PCR programme, the samples were compared by electrophoresis of $10-\mu \mathrm{l}$ samples in agarose $1.5 \%$ gels in Tris-acetate buffer $(0.04 \mathrm{M}$ Trisacetate, $0.002 \mathrm{M}$ EDTA, pH 8.5) stained with ethidium bromide and photographed on a UV light transilluminator by the Polaroid MP4 system. A standard 1-kb DNA ladder (Gibco BRL, Burlington, Canada) was included.

\section{rep-PCR products analysis}

Comparisons between the fingerprints were performed as described previously [18]. B riefly, computer-assisted analysis of the scanned fingerprint was performed with

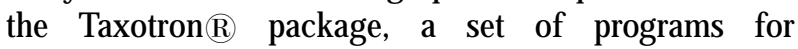
molecular systematics [19]. Only major bands were considered and band intensity was not used as a criterion. The derived molecular sizes (in bp) were used to compute distance (D) based upon band sharing as the complement of the Dice coefficient [20]: $D=1-\left(2 n_{x y} / n_{x}+n_{y}\right)$; where $n_{x y}$ is the number of bands in each strain; $n_{x}$ and $n_{y}$ are numbers of total bands scored for each individual. In the pairwise comparison to match co-migrating fragment positions between pairs of rep-PCR, a match was recorded if the normalised molecular size of the first amplicon was within a window of $3 \%$ of the molecular size of the second amplicon. A matrix combining all the distances was generated from which cluster analysis was performed by the unweighted pair-group method with mathematic averages (UPGMA) and a dendrogram was constructed.

\section{Results}

The reproducibility of both methods was tested with DNA preparations made from separate cultures on different days and identical and reproducible strain-

Table 1. B. fragilis strains analysed

\begin{tabular}{|c|c|}
\hline Strains & Source \\
\hline ATCC 23745, ATCC 25285 & $\begin{array}{l}\text { A merican Type Culture Collection (ATCC) Rockville, } \\
\text { MD, USA }\end{array}$ \\
\hline $016 \mathrm{M}, 022 \mathrm{H}, 023 \mathrm{~A}$ & Otitis media \\
\hline $1031,1033^{*}, 1048-a, 1058-1,1304-2,1034$ & Intra-abdominal infections \\
\hline $1032,1037,1039,1081,1241$ & B acteraemia \\
\hline M C2, M C3, 048203, M C1, 1292, 1077 & Soft tissue infections \\
\hline $077225-2^{\dagger}, 078819-3^{\dagger}, 2832.1^{\dagger}, 20656.2 .1^{\dagger}$, FD $10 B 1,079298-3^{\dagger}$ & Human intestinal infection \\
\hline FF10, FF14, FF15, F36-6, F43-6 & Human intestinal microflora \\
\hline AA 1, AA3, AA7, AA 10*, AA41-1, AA42-1, AA42-2, AAa & A quatic environment \\
\hline
\end{tabular}

*The cfiA gene was detected in strains AA10 and 1033, but not in the other strains (unpublished observations).

†Strains given by Dr Lyle Myers, M ontana University, EUA. 
specific patterns were obtained. Informative and reproducible fingerprints were obtained with $2.5 \mathrm{U}$ of Taq polymerase. Higher concentrations of Taq polymerase resulted in a higher background without additional prominent bands (data not shown). A concentration of $50 \mathrm{ng}$ in each reaction was used throughout this study.

To investigate the genetic relationship among the $41 \mathrm{~B}$. fragilis strains, the profiles of each sample were determined by rep-PCR with REP and ERIC primers. In both methods, amplification reactions generated informative arrays of bands composed of a minimum of three and a maximum of 16 bands with $\mathrm{M}_{\mathrm{r}} \mathrm{s}$ ranging from 100 to c. $2200 \mathrm{bp}$.

A mplification of the $41 \mathrm{~B}$. fragilis isolates with REP primer resulted in three-to-seven amplicons located between the 200-bp and 2200-bp markers of the DNA ladder (Fig. 1). ERIC primers revealed 11-16 amplicons ranging from $100 \mathrm{bp}$ to $2200 \mathrm{bp}$ (Fig. 2).

The combined data from the two consensus sequences (REP and ERIC) were compiled and a dendrogram was generated (Fig. 3). When a distance of 0.30 was used as a threshold, three clusters (hereafter referred to as genotypes I, II and III) were generated. Genotype I included 37 strains of different origins and it was subdivided into two other groups, genotype IA and IB, with a genetic distance of 0.22 . Genotype IA included 18 strains, of which four were isolated from intraabdominal infections, two from intestinal microflora,

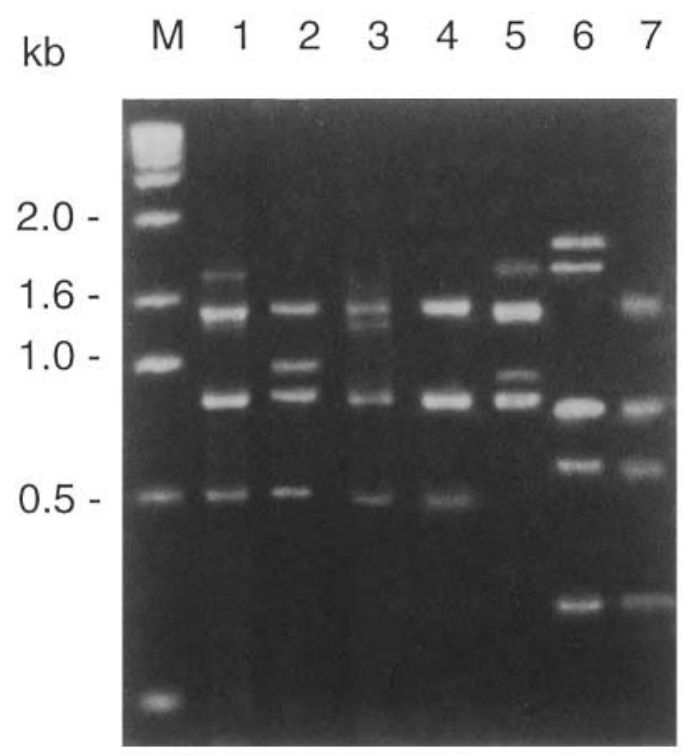

Fig. 1. rep-PCR fingerprints of $B$. fragilis isolates obtained with primer REP. Lane M, 1-kb DNA ladder; 1, enterotoxigenic strain 20656.2.1 (genotype IA); 2, nonenterotoxigenic strain 077225-2 (genotype IA); 3, ATCC 25285 (genotype IB); 4, ATCC 23745 (genotype IB); 5 , strain AA1 (genotype II); 6, strain 1033 (genotype III); 7, strain FD 10 B 1 (genotype III). The cfiA gene was detected in strain 1033.

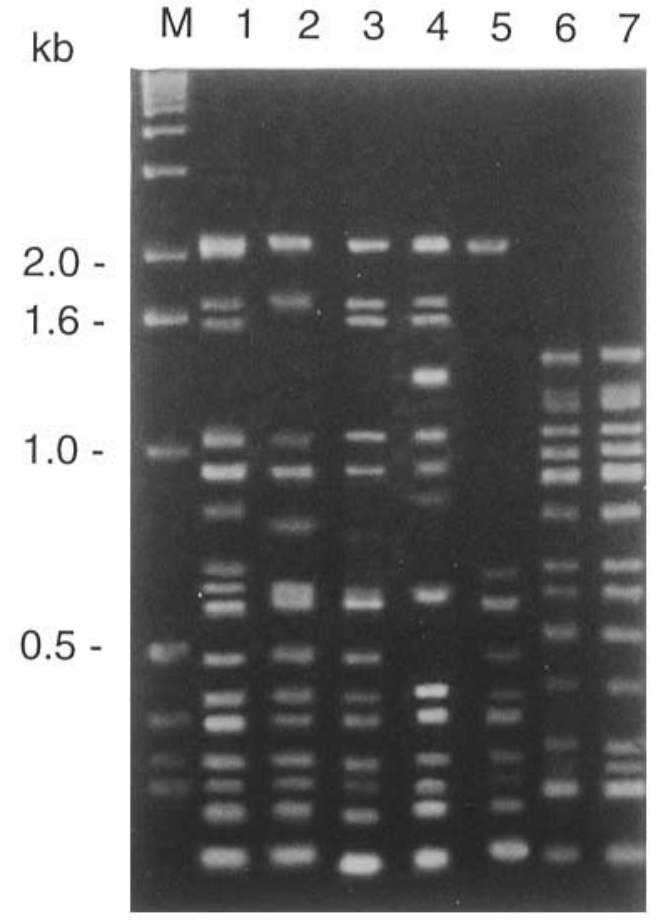

Fig. 2. rep-PCR fingerprints of $B$. fragilis isolates obtained with primer ERIC. Lane M, 1-kb DNA ladder; 1, enterotoxigenic strain 20656.2.1 (genotype IA); 2, nonenterotoxigenic strain 077225-2 (genotype IB); 3, ATCC 25285 (genotype IB); 4, ATCC 23745 (genotype IB); 5, strain AA1 (genotype II); 6, strain 1033 (genotype III); 7, strain FD 10 B 1 (genotype III). The cfiA gene was detected in strain 1033.

five from intestinal infections, one from otitis media and six from an aquatic environment. Genotype IB included 19 strains, of which 14 were isolated from non-intestinal infections (five from bacteraemia, four from intra-abdominal infection, three from soft tissue infection and two from otitis media), three from intestinal microflora and the two reference strains. Genotype II consisted of two strains from an aquatic environment (AAa and $A A 1$ ) and genotype III consisted of two strains, of which one was isolated from intra-abdominal infection (1033) and one from intestinal infection (FD 10B1). The average genetic distances among these groups were as follows: 0.31 between groups I and II and 0.48 between groups I, II and III.

\section{Discussion}

This study demonstrated that repetitive extragenic sequences such as REP and ERIC are present in the genome of diverse $B$. fragilis strains, confirming and extending the suggestion made by other authors that these sequences are virtually ubiquitous $[14,21]$. The results also suggest that ERIC-like sequences are more widely distributed in the $B$. fragilis genome than REPlike sequences. How this fact can benefit $B$. fragilis has not yet been determined. However, their presence and 


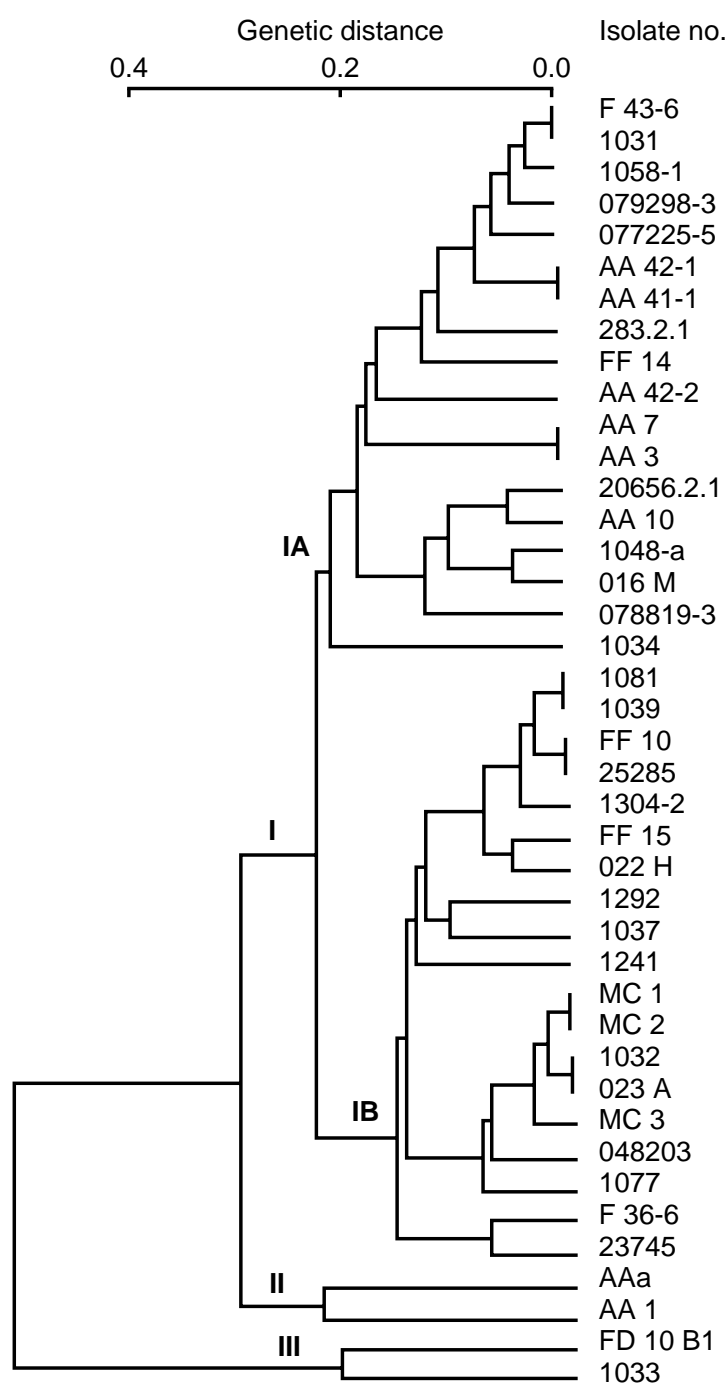

Source

Human intestinal microflora Intra-abdominal infection Intra-abdominal infection Human intestinal infection Human intestinal infection Aquatic environmental Aquatic environmental Human intestinal infection Human intestinal microflora Aquatic environmental Aquatic environmental Aquatic environmental Human intestinal infection Aquatic environmental Intra-abdominal infection Otitis media

Human intestinal infection Intra-abdominal infection Bacteraemia

Bacteraemia

Human intestinal microflora ATCC

Intra-abdominal infection Human intestinal microflora Otitis media

Soft tissue infection

Bacteraemia

Bacteraemia

Soft tissue infection

Soft tissue infection

Bacteraemia

Otitis media

Soft tissue infection

Soft tissue infection

Soft tissue infection

Human intestinal microflora ATCC

Aquatic environmental Aquatic environmental Human intestinal infection Intra-abdominal infection

Fig. 3. Dendrogram generated by combining the rep-PCR fingerprints obtained with each of the two primers $R E P$ and ERIC. The genetic relatedness of $41 \mathrm{~B}$. fragilis isolates can be resolved at a distance of 0.30 as three clusters (I, II and III) defining three genotypes. The cfiA gene was detected in strains AA 10 and 1033, but not in the other strains (unpublished observations). Strains 077225-2, 078819-3, 283.2.1 (non-enteroxigenic) and 20656.2.1, 079298-3 (enterotoxigenic) were given by Dr L. Myers, M ontana University, EUA.

widespread distribution in both prokaryotic and eukaryotic genomes strongly suggests that they are important to the structure and evolution of genomes [14, 21, 22]. In spite of the fact that the precise functions of repetitive sequences in prokaryotic genomes are still obscure, their presence can be used both in applications and molecular genetic manipulations. In recent years, some studies have demonstrated that the REP- and ERIC-PCR protocols, referred to collectively as rep$P C R$, are particularly suitable for the rapid molecular characterisation of bacterial species.

This molecular approach has been accepted as a valuable and simple alternative to other typing methods. In the present study, reproducible fingerprint profiles of a particular $B$. fragilis strain could be generated from DNA isolated at different times from the same colony, from different colonies of the same strain, or from serial cultures of the same strain. This suggests that DNA fingerprinting generated by rep-PCR constitutes a powerful tool for studying B. fragilis, as well as other bacterial pathogens.

As B. fragilis isolates were obtained from diverse biological origins, the samples can be regarded as representative of the natural population of the taxon. The diversity of the taxon $B$. fragilis has already been demonstrated by genotypic and phenotypic methods. Other studies, using REA and RFLP $[6,7]$ and APPCR [9], clearly demonstrated a wide genetic diversity within the species B. fragilis, consisting of nearly as many genotypes as there were isolates. In the present study, the use of a computer-assisted system to analyse the results confirmed a reasonable $B$. fragilis diversity by rep-PCR.

The polymorphism obtained with ERIC and REP allowed the $41 \mathrm{~B}$. fragilis isolates to be distributed into a dendogram in three genotypes (genotype I, II and III) based upon a calculated genetic distance (Fig. 
3). Genotype I constituted the most heterogeneous cluster in relation to the biological origin and represented the majority of the strains in this study. The strains of this cluster, isolated from different types of clinical sample (faeces, blood, suppuration, etc.) and from aquatic environments, showed an overall similarity of $70 \%$. Cluster IA was formed by the majority of the strains obtained from infectious processes in the intestinal mucosa area, i.e., strains from human intraabdominal infections and intestinal microflora or from aquatic environment - except for strain 016M which was isolated from a case of otitis media. Two enterotoxigenic strains (079298-3 and 20656.2.1) and three non-enterotoxigenic strains $(077225-5,283.2 .1$ and 078819-3; kindly given by $\mathrm{Dr}$ L. M yers, Montana University, EUA), were included in this group. The strains in this cluster showed genetic similarity of $c$. $80 \%$. It appears that it was not possible to observe distinct groups of $\mathrm{Ent}^{+}$and $\mathrm{Ent}^{-}$strains by means of rep-PCR methods. On the other hand, cluster IB consisted of the majority of strains isolated from infectious processes far from the intestinal mucosa area (bacteraemias, otitis media, etc.), which showed genetic similarity of $85 \%$. Based on this fact, different types could cohabit in intestinal microflora and be responsible for causing infectious processes. Strain 016M, isolated from otitis media and grouped in cluster IA, could have originated from intestinal microflora by contact with contaminated objects or hands, thus allowing colonisation of the ear. The relationship between mucosal nasopharynx microflora and otitis media caused by $B$. fragilis has not been confirmed yet.

Genotype II, composed of two strains isolated from an aquatic environment, showed genetic similarity of $79 \%$. The possibility of contamination with animal faeces could explain this finding. However, complementary studies would be needed to determine whether there is truly a relationship between strains of $B$. fragilis isolated from human intestinal microflora and strains from animal intestinal microflora.

Genotype III showed more genetic distance in this study; it grouped the strains into a genetic distance of 0.5 . However, the genetic similarity between the strains in this group was up to $80 \%$. Could these strains constitute a new genospecies? Several studies have shown rep-PCR to have a good correlation with pulsedfield gel electrophoresis (PFGE) results. In spite of these facts, the strains are phenotypically very homogeneous [23]. Other studies are needed to confirm if important phenotypic traits should be superimposed upon the polymorphism results to justify species designations [24].

Two $\beta$-lactamases, the endogenous cephalosporinase CepA and the carbapenemase CfiA, appear to be confined to the species $B$. fragilis. Furthermore, as suggested by Podglajen et al. [8], cfiA-positive strains may represent a novel species. The use of PCR with specific primers has shown that the cfiA gene exists in strains AA10 (isolated from an aquatic environment) and 1033 (isolated from an intra-abdominal infection) (unpublished observations). However, these strains could not be found in the same genotype based on the results obtained by combined data from the two primers.

In spite of the wide diversity of the strains studied, including human (clinical and non-clinical) or environmental sources and geographic origin, it was not possible to detect an obvious correlation between a given genotype and the specific disease it can cause or healthy state of the host, or even the polluted environment where it can be found. Nevertheless, the $P C R$ fingerprint techniques used here proved to be relatively simple to perform and reliable for $B$. fragilis genetic diversity studies.

We thank Walter Oellemann for constructive criticism and Joaquim Santos Filho for technical assistance. This work was supported by grants from the following institutions: CNPq, PRONEX, FAPERJ, CAPES, FUJB, FINEP and CEPG.

\section{Refer ences}

1. Gorbach SL, Bartlett JG. A naerobic infectious. N Engl Med 1974; 290: 1177-1184.

2. Polk $B F, K$ asper $D L$. Bacteroides fragilis subspecies in clinical isolates. Ann Intern Med 1977; 86: 569-571.

3. Myers $L L$, Shoop DS, Stackhouse $L L$ et al. Isolation of enterotoxigenic Bacteroides fragilis from humans with diarrhea. J Clin Microbiol 1987; 25: 2330-2333.

4. Sack BS, Albert MJ, Alam K, Neogi PKB, Akbar MS. Isolation of enterotoxigenic Bacteroides fragilis from $B$ angladeshi children with diarrhea: a controlled study. J Clin Microbiol 1994; 32: 9600-963.

5. Pantosti A, Colangeli R, Tzianabos AO, Kasper DL. Monoclonal antibodies to detect capsular diversity among Bacteroides fragilis isolates. J Clin M icrobiol 1995; 33: 2647-2652.

6. Smith CJ, Callihan DR. A nalysis of rRNA restriction fragment length polymorphisms from Bacteroides spp. and Bacteroides fragilis isolates associated with diarrhea in humans and animals. J Clin Microbiol 1992; 30: 806-812.

7. Kleivdal $\mathrm{H}$, Hofstad T. Chromosomal restriction endonuclease analysis and ribotyping of Bacteroides fragilis. APMIS 1995; 103: $180-184$.

8. Podglajen I, Breuil J, Casin I, Collatz E. Genotypic identification of two groups within the species Bacteroides fragilis by ribotyping and by analysis of PCR-generated fragment patterns and insertion sequence content. J Bacteriol 1995; 177: $5270-5275$.

9. M oraes SR, Gonçalves RB, Mouton C, Seldin L, Ferreira MCS, Domingues RMCP. Bacteroides fragilis isolates compared by AP-PCR. Res Microbiol 1999; 150: 257-263.

10. Lin AW, Usera MA, Barrett TJ, Goldsby RA. Application of random amplified polymorphic DNA analysis to differentiate strains of Salmonella enteritidis. J Clin Microbiol 1996; 34: 870-876.

11. M énard C, Mouton C. Clonal diversity of the taxon Porphyromonas gingivalis assessed by random amplified polymorphic DNA fingerprinting. Infect Immun 1995; 63: 2522- 2531

12. de Bruijn FJ. Use of repetitive (repetitive extragenic palindromic and enterobacterial repetitive intergeneric consensus) sequences and the polymerase chain reaction to fingerprint the genomes of Rhizobium meliloti isolates and other soil bacteria. Appl Environ Microbiol 1992; 58: 2180-2187.

13. Dimri GP, Rudd KE, Morgan MK, Bayat $H$, A mes GF-L. Physical mapping of repetitive extragenic palindromic sequences in Escherichia coli and phylogenetic distribution 
among Escherichia coli strains and other enteric bacteria. J Bacteriol 1992; 174: 4583-4593.

14. Versalovic J, Koeuth T, Lupski JR. Distribution of repetitive DNA sequences in eubacteria and application to fingerprinting of bacterial genomes. Nucleic Acids Res 1991; 19: 6823-6831.

15. Summanen $\mathrm{P}, \mathrm{B}$ aron $E$, Citron DM, Strong C, Wexler HM, Finegold SM. Wadsworth Anaerobic Bacteriology Manual. Belmont, CA, Star Publishing Company. 1993.

16. Smith G, Socransky S, Smith C. Rapid method for the purification of DNA from subgingival microorganisms. Oral Microbiol Immunol 1989; 4: 47-51.

17. Wilson K. Preparation of genomic DNA from bacteria. In: A usubel FM, B rent R, Kingston RE, Moore DD, Seidman JG, Struhl $\mathrm{K}$ (eds) Current protocols in molecular biology. New York, Greene Pub. A ssociates and Wiley-Interscience. 1991: 2.4.12.4.2.

18. Paquet $C$, Mouton $C$. RAPD fingerprint for the distinction of Prevotella intermedia sensu stricto from Prevotella nigrescens. Anaerobe 1997; 3: 271-278.
19. Grimont PAD. Taxotron package: User's manual. Paris, Institute Pasteur. 1994.

20. Dice LR. Measures of the amount of ecologic association between species. Ecology 1945; 26: 297-302.

21. Louws FJ, Fulbright DW, Stephens CT, de Bruijn FJ. Specific genomic fingerprints of phytopathogenic Xanthomonas and Pseudomonas pathovars and strains generated with repetitive sequences and PCR. Appl Environ Microbiol 1994; 60: 2286-2295.

22. Lupski JR, Weinstock GM. Short, interspersed repetitive DNA sequences in prokaryotic genomes. I Bacteriol 1992; 174: $4525-4529$

23. Olive DM, Bean P. Principles and applications of methods for DNA-based typing of microbial organisms. J Clin Microbiol 1999; 37: 1661-1669.

24. Johnson JL. Specific strains of Bacteroides species in human fecal flora as measured by deoxyribonucleic acid homology. Appl Environ Microbiol 1980; 39: 407-413. 